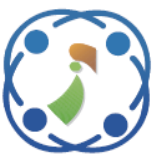

\title{
Weights-Based Energy-Efficient Wireless Sensor Network Protocol with Firefly Synchronization for Illegal Logging
}

\author{
Giva Andriana Mutiara ${ }^{1 *}$ \\ Othman Mohd ${ }^{2}$ \\ Nanna Suryana ${ }^{2}$ \\ Ahmad Naim Che Pee ${ }^{2}$ \\ ${ }^{1}$ Applied Sciences School, Telkom University, Indonesia \\ ${ }^{2}$ Faculty of Information and Communication Technology, Universiti Teknikal Malaysia Melaka, Malaysia \\ *Corresponding author's Email: givamz@telkomuniversity.ac.id
}

\begin{abstract}
An effort to minimize illegal logging by utilizing advanced technology such as Wireless Sensor Network (WSN) are highly required. WSN is a communication solution between remote sensors located in isolated wide forest areas. However, the WSN challenge in a remote and wide-area has the energy resources limitation. This research proposed a weights-based energy-efficient WSN data communication protocol with firefly synchronization, namely Firefly Synchronization Multi-hop - Geographical Energy-Aware Routing (FSM-GEAR) Protocol. This protocol aimed to increase the network lifetime of each node by shortening the transmission distance. Firefly synchronization between sensors is conducted to reduce the data transmission waiting time. A weights-based approach in cluster head $(\mathrm{CH})$ selection phases and energy consumption analysis are formulated according to the comparison between nearest distance to Gateway and the maximum residual energy of each node based on received signal strength indicator (RSSI). Weights-based FSM-GEAR performance analysis is carried out based on the parameters of network lifetime, residual energy, and throughput with the Modified-GEAR and enhanced Modified-GEAR protocols as a comparison. The simulation result stated that FSM-GEAR protocol showed better network lifetime, residual energy, and throughput than both protocols.
\end{abstract}

Keywords: Weights-based energy-efficient protocol, Firefly synchronization, Illegal logging, Wireless sensor network, Network lifetime, Geographical energy-aware routing.

\section{Introduction}

Wireless Sensor Networks (WSN) suit technology for the outdoor application, which has wide area supervised environment that mostly inaccessible by the human, and for long-term monitoring application [1]. Besides, the well-planned WSN that is deployed in outdoor environments offers a decentralized data collection that is reliable, costefficient, and minimal human intervention [2].

WSNs consist of many sensor nodes (it can be more than hundreds) of integrated small hardware that can communicate with limited power [3]. The integration of sensors into a wireless network is closely associated with the physical environment and perceives various environmental effects to form an embedded sensor device. Sensors devices integrated with processing circuit, a transmitter and receiver communication devices, and limited power of the battery is called as Micro Electro Mechanical System (MEMS). Therefore, WSNs are determined as an adhoc network that consists of a lot of MEMS, which has limited energy capacity to sense and monitor the environment [4].

Currently, deforestation has happened in many countries, even in the countries located in Southeast Asia. Deforestation will threaten the forest ecosystem and also human life. The effects of deforestation are global warming, disruption of the global water cycle, decreased biodiversity, habitat loss and conflict, economic losses, and social consequences [5].

Illegal logging becomes the most significant issue of deforestation occurring in Indonesia [6]. Illegal logging is determined as an activity caused by logging the trees without any permission. Indirect impacts of deforestation by illegal logging have affected the economic domain. The Indonesian 
government itself has lost its income by billions of dollars.

There have been various technologies and methods of problem-solving implemented to tackle illegal logging in many countries. However, WSN is still the most appropriate technology that can be applied to tackle it [7]. WSN can fulfil the forest characteristics located in outdoor circumstances, vast and remote areas, and hardly access by humans.

Several aspects must be considered in the WSN, such as limited memory, small computational ability, the strength of the wireless signal sensor, manageable sensor, synchronization, localization, routing protocols, high data rate, interoperability, energyefficiency, limited and non-rechargeable batteries [8]. Based on these considerations, the WSN-based application must be less maintenance and robust. The performance of the WSN is improved by increasing the network lifetime [9]. Network lifetime becomes the most considered aspect for viability since it is one of the parameters determining how long the system can work appropriately without annual maintenances.

The network lifetime is affected by power consumption [10]. Since the MEMs is power limited, the controlling of power consumption must be optimized. $80 \%$ of power consumption is used for the communication process [11]. The communication process is not only transmitting and receiving data but also the routing setting to manage the communication process in the network. Thus, the communication process is affected by the localization technique, network architecture such as single-hop or multi-hop network, routing protocol and clustering, and synchronization. Single-hop network applied in small area sensing, while multi-hop network applied in wide area sensing and support scalability [12].

Illegal logging WSN-based application is suitable for location-based routing protocol network structure, which concerns the geographical location-based information of all the sensor nodes. Geographic and Energy Aware Routing (GEAR) is applied for illegal logging application to transmit the data packet. In this protocol, Gateway (GW) is installed at the middle location between the Base Station (BS) and the sensor node $(\mathrm{SN})$ to ease the communication process and to increase the network lifetime [13].

Multi-hop GEAR (M-GEAR) WSN provides scalability that usually consists of a high-density sensor node and creates short-distance communication to have the most extended lifetime and reduce energy consumption. Multi-hop structure network gives challenges in manageable the cluster formation and cluster head $(\mathrm{CH})$ selection.

The synchronization of the SNs also gives the challenge to be enhanced to minimize waiting time and reduce power consumption in the communication process. Thus, clock synchronization is essential to make an optimized scheduling clock. Firefly based universal synchronization algorithm (FUSA) is an algorithm based on fireflies modelled that multipurpose regardless of the network topology. This algorithm is high-fully distributed cooperation and suitable for the multi-hop network. The blink of light in fireflies is modelled as synchronized when the clock's frequency is perfectly synchronized, and phases differ [14].

The motivation for body of knowledge comes from the fact that the clustering protocol and synchronization network consume less energy. However, the existing clustering has significant drawbacks such as randomize nodes become the $\mathrm{CH}$, unequal distribution of energy consumption in one cluster and continuously sending data even though there are no information changes. Those drawbacks are dropping the inefficient consumption of energy [3]. In the multiple level schemes, it cannot overcome the $\mathrm{CH}$ located far away from the BS [15]. Meanwhile, the scheduling using TDMA also need to be optimized to reduce the power consumption.

This research was aimed to optimize network performance in the routing protocol. The research focused on the WSN infrastructure that suits to illegal logging tracking application, clustering method, routing protocol, and synchronization algorithm without considering security aspects. The main contribution of this research is a new geographical routing protocol called Firefly Synchronization Multi-hop Geographic Energy-Aware Routing (FSM-GEAR) that considering the weights-based parameter. Weights-based parameter is proposed by considering the nearest distance parameter, the highest remaining energy, and the minimum power consumption as parameters that are owned by SNs.

The simulation results provided used the MATLAB. The simulation testing of the proposed protocol is able to optimize the network performances in metrics network lifetime, residual energy, and throughput. The power evenly distributes and improves the network performance to expand the network lifetime by reducing power consumption.

The rest of the chapters in this paper is outlined as follows: In Section 2, the related work is presented and discussed. Next in Section 3, FSM-GEAR is described as a proposed algorithm in the step of phases. In Section 4, the simulation and the result of the proposed system based on MATLAB are used to test the performance using several scenarios. The validation results were discussed and analysed. Section 5 is outlined the summary of the conclusion and the future work. 


\section{Related works}

This section describes related works that support and influence the research. It was included on how WSN implemented in illegal logging area, what is the challenges in WSN domain, and the appropriate network for illegal logging.

First of all, the first study of related works is discussing about the development of the technology supporting the illegal logging domain. There were various approached techniques and methods used to tackle illegal logging. The previous technique was using the Radio Frequency Identification (RFID) and the reader [16]. RFID could track and identify the trees and logs [17]. But unfortunately, RFID has a drawback in the limited coverage area.

A new development in the wireless method in illegal logging domain was introduced by S.Wang [18] taking advantages of Received Signal Strength Indicator (RSSI) distance coverage when communicating in the WSN using GPRS. It analyzes the received wireless signal strength between sensor nodes. The change of the trunk position can detect as the falling down trees. Unfortunately, the GPRS is not supported the connected in the wide-forest.

Another effort has been proposed by Al-Turjman [19], which explored how to model the optimal connectivity for WSN in the forest. Since the forestry environment is harsh and risk, it is required an optimal solution to locate the relay node in $3 \mathrm{D}$ forestry space. Unfortunately, this research needs more exploration under further constraints, such as coverage, routing protocol, synchronization, energy consumption, and data fidelity. According to those related works, there is a challenge to improve the sensor nodes distribution based on RSSI distance coverage.

Meanwhile, as mention in the introduction section, illegal logging application was appropriate with GEAR protocol. GEAR protocol is included in location-based routing protocols for WSN. Yu [20], introduced GEAR using energy-aware neighbor selection to route a packet in the networks. The sensors are equipped with localization hardware. Thus, they are aware of their current position. Furthermore, the sensor also aware of its residual energy. Therefore, GEAR selects the routing path by referring to the node's location, shortening the transmission distances of the sensor nodes, and extending the network lifetime [21].

Q.Nadeem presented the gateway-based energyaware multi-hop routing protocol to handle the remote sensor nodes when transmitting data to BS. This routing is called modified GEAR or M-GEAR. The M-GEAR aims to optimize the energy consumption and network lifetime. The analysis parameters are network lifetime, residual energy, and throughput. The network structure is divided into eight logical regions, six clusters with a $\mathrm{CH}$ for each cluster. The gateway is installed in the sensing area center, while the BS itself is installed out of the sensing area [15]. The sensor nodes were organized by TDMA scheduling.

N.Singh [22] enhanced M-GEAR that focuses on clustering techniques by distance and the maximum energy of nodes. The clustering focuses on distancebased cluster head selection and load-balancing from higher energy in the cluster. BS is installed outside the sensor field, and the gateway is installed in the middle of the sensor field. The network is divided into four regions. The simulation result increases the network lifetime regarding M-GEAR.

According to the related works of GEAR protocol, the development and modification are carried out on the number of clusters and gateways. All the modification is an effort to have low-power consumption in the network. Thus, it is become the second challenge to modify and enhance the GEAR protocol configuration on the number of clusters, $\mathrm{CH}$ selection method, and gateways in order to have a low-power protocol.

The last effort to have a low-power protocol is clock-synchronization [23]. Clock-synchronization is a crucial factor for distributing sensor nodes in the network. Routing protocol, communication between nodes and BS in the network, and synchronization algorithm are engaged in the WSN. The stable integration can enhance network performance and reduce power consumption.

A new clustering approached called Firefly-based clustering approach (FiCA) and Spatial Fireflybased clustering approach (SFiCA) was suggested by N. Jabeur [24]. The result showed that some performances required to be fixed but gave a promising result in terms of cluster distribution. Firefly Algorithm was also propounded for synchronization in clustering protocol in the WSN by E. Kumar [25] and M. Baskaran [26]. The firefly synchronization was applied in the hierarchical WSN network, low-power energy adaptive clustering hierarchy (LEACH). Fire-LEACH aimed to reduce energy consumption and increase network throughput by reducing delay in a packet transfer. The algorithm was compared to LEACH, the simulation result proved that the implementation of firefly synchronization at LEACH protocol could improve the network performance.

According to those related works, Firefly algorithm offers the third potential improvement to the proposed research. Therefore, the firefly 
algorithm can be adapted to reduce the transmission waiting time and arrange the scheduling transmission.

\section{Proposed weights-based FSM-GEAR protocol}

This section describes how the weights-based FSM-GEAR protocol is designed for illegal logging. This protocol is proposed considering the challenging to apply low-power WSN for illegal logging application. Because the use of WSN is getting higher, the challenge is an effort to reduce power consumption.

The first potential improvement is SNs distributed. The SNs distribution is allocated by the range of RSSI in the simulation. The second potential improvement is the power parameter, influenced by the appropriate network structure for illegal logging, the data exchange protocol as a routing protocol to support communication between SNs, GW, BS, scheduling, and synchronization network.

For the second potential improvement, FSMGEAR protocol designs the inter-node coordination rules to improve the selection and optimization of routing paths in various sensor nodes based on weight-based parameter. Weights-based parameter is used considering the nearest distance parameter, the highest remaining energy, and the minimum power consumption as parameters that are owned by SNs. Weights-based parameter are applied in the $\mathrm{CH}$ Selection phase and energy performance parameter.

The third potential improvement is applying the Firefly algorithm that conducts the optimization to synchronize the convergence clock at each node. Optimizing the routing path provides more low overhead and longer route lifetime. Besides, the firefly algorithm will decide whether a particular sensor node can transmit the data packet. This function acts as scheduling in the data packet transmission.

Thus, based on those potential improvements, this proposed system aimed and focused on having a low-power consumption based on illegal logging model application, including how the sensor node is distributed in the network, multi-hop GEAR protocol implementation, clustering, $\mathrm{CH}$ selection mechanism, and implemented Firefly algorithm as a synchronization protocol that supports data transmission scheduling in the network.

\subsection{Establishment network topology}

The proposed low-power protocol network topology is established according to the M-GEAR

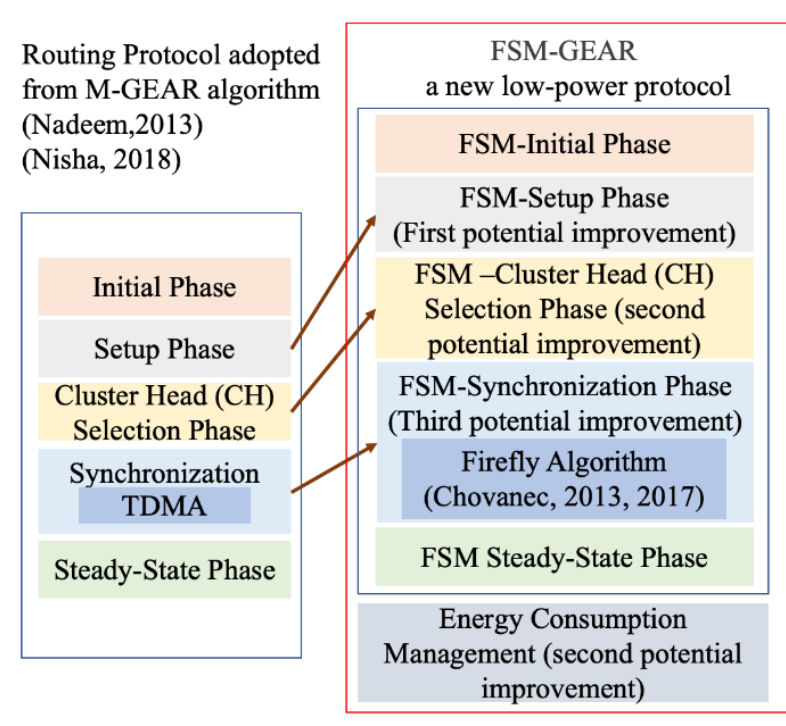

Figure. 1 FSM-GEAR Phases

phases. As seen in Fig. 1, the phases are consisted of five phases. M-GEAR was adapted from Q.Nadeem [13], and enhanced M-GEAR from N.Singh [22]; meanwhile, Firefly was from Chovanec [23].

Generally, M-GEAR network that proposed by Q.Nadeem has the same region composition with N.Singh. It was region near to BS, region near to $\mathrm{GW}$, and clustered region that formed a $\mathrm{CH}$ selection. Both, was using one GW in their network. The differences were come from the used parameters. Q.Nadeem used probability and threshold to select the first $\mathrm{CH}$ and considering the remaining energy for next $\mathrm{CH}$. While N.Singh stated the parameter distance and maximum energy in the research. The distance parameter is used to determine the regions, and the maximum energy is used to select the $\mathrm{CH}$.

According to Fig. 1, FSM-GEAR Phases was established based on illegal logging requirements. Thus, all phases of the new low-power protocol were also modified as illegal logging requirements based on all potential improvements. The modification is on the protocol and network model that suit to the illegal logging domain. The detailed explanation will be described in the next subchapter sequentially.

In Fig. 2, FSM-GEAR phases are detailed in several algorithms and mechanisms. FSM-Initial Phase and FSM-Setup phase are configured at BS. While FSM-Cluster head selection phase, FSMSynchronization phase and FSM-Steady State Phase are configured at cluster head and sensor nodes. At the FSM-initial phase, the sensor node is distributed in random coordinate using modified 2D Gaussian Distribution as formulated in Eq. (1). In the state of the setup phase, the sensor nodes distribution algorithm is introduced based on the RSSI signal 


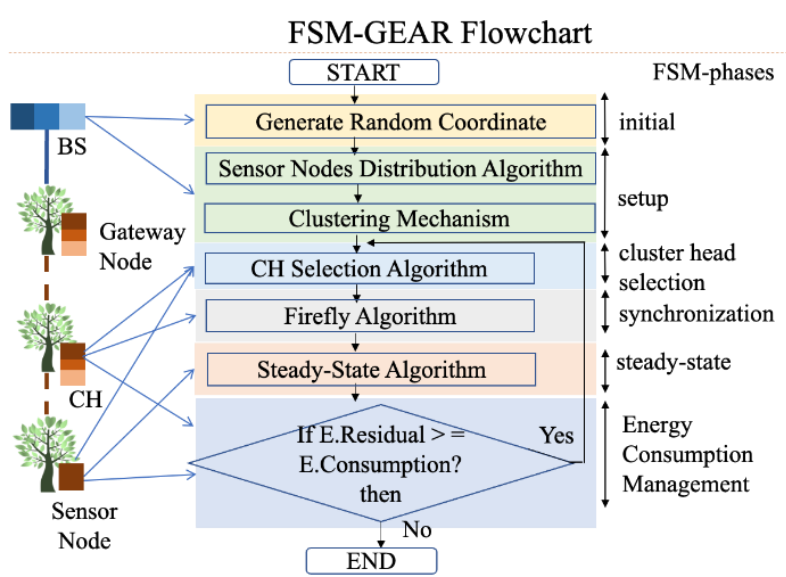

Figure. 2 FSM-GEAR Flowchart

distance of the hardware radio communication. This algorithm divides the sensor node into several regions based on their connection to BS, GW, or cluster. The clustering formation in the network is introduced using a clustering mechanism based on the distance and the location.

FSM-cluster head selection phase is introduced based on weights-based parameter consists of distance between sensor nodes to GW and maximum residual energy. Once the cluster head has been defined, the next state is a node and network synchronization with Firefly algorithm adaption. In this phase, sensor nodes transmit data packets once its clock reaches a maximum value as the firefly algorithm. This mechanism shows the firefly algorithm acting as a data transmission scheduling as well as TDMA in GEAR. After all sensor nodes have been transmitted to the data packets, all sensor nodes are in sleep mode at the steady-state phase. If any sensor nodes have enough energy residual to transmit the data packets, they are back to the $\mathrm{CH}$ selection phase and continue to synchronization phase. These circumstances will be continued until all sensor nodes are depleted.

\subsubsection{FSM initial state}

At this phase, 100 sensor nodes were distributed in the 100x100 sensor field region at the first quadrant in the cartesian graph. Sensor nodes were distributed using modified 2D Elliptical Gaussian [27] as stated in the Eq. (1). In Eq. (1) $\left(a_{0}, b_{0}\right)$ denotes the sensor nodes position. While $\sigma_{a}$ and $\sigma_{b}$ are denotes as standard deviations for $a$ and $b$ dimensions, respectively.

The modified were limited due to the sensor nodes region coverage, must be at a maximum twice distance range of RSSI. The BS was located in the coordinate [0.0]. While the sensor nodes had been

$$
\begin{gathered}
f(a, b)=\frac{1}{2 \pi \sigma_{a} \sigma_{b}} \exp -\left(\frac{\left(a-a_{0}\right)^{2}}{2 \sigma_{a}^{2}}\right. \\
\left.+\frac{\left(b-b_{0}\right)^{2}}{2 \sigma_{b}^{2}}\right)
\end{gathered}
$$

distributed, GW was located in the middle of the sensor field based on RSSI distance. The mapping of distribution sensor node as FSM-GEAR model network can be seen in Fig. 3. The sensor nodes were scattered in three regions; $\mathrm{BS}$ region, $\mathrm{GW}$ region, and cluster region. The classification of sensor nodes will be defined in the FSM setup phase.

\subsubsection{FSM setup state}

After distributed sensor nodes at the initial phase has been defined, the sensor nodes will be divided

into region classification at FSM-Setup phase, which consists of sensor nodes distribution algorithm and clustering mechanism, as a phase to organize network configuration. The classification is determined as seen in Eq. (2).

Sensor nodes distribution algorithm determined by regions of sensor nodes in the network is classified in three categories: BS region, GW region, and cluster region as formulated in Eq. (2) and Fig. 4. Based on Eq. (2), $d_{B}$ is stated as distance to BS, $d_{G}$ is defined as distance to $\mathrm{GW}$, and $d_{S}$ is defined as distance among sensor nodes. The distance is measured by RSSI metric. Then, proceed to mapping

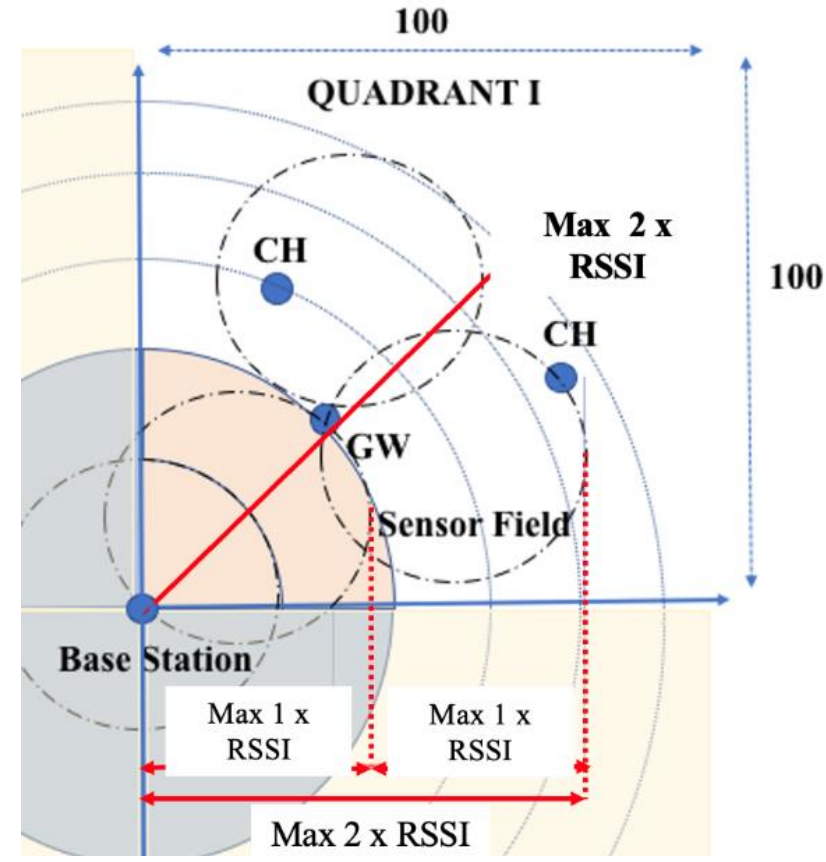

Figure. 3 FSM-GEAR model network 


$$
\begin{aligned}
& \operatorname{Reg}(n)
\end{aligned}
$$

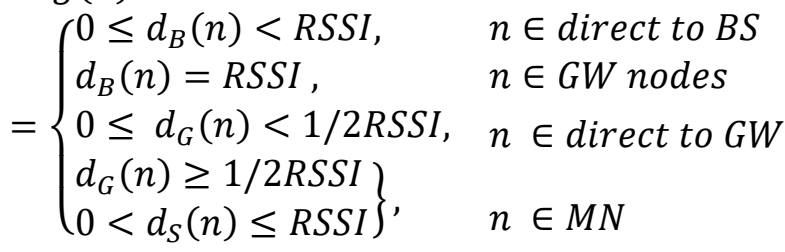

sensor nodes on the flowchart in Fig. 4. BS region is the zone where the sensor node is located near to BS, in maximum range zero until one RSSI distance. All the sensor nodes in this region use a direct communication to transmit their data to BS. GW region is a sensor node located near $\mathrm{GW}$, ranging from 0 to a half RSSI distance from GW. All the sensor nodes in this region transmit the data directly to GW then continue from GW to BS. GW itself is located in one RSSI distance from BS. The rest of the sensor nodes, which has a distance more than a half RSSI to GW are determined as cluster region. The distance among sensor nodes also determined in range 0 to one RSSI distance as cluster member nodes.

In cluster region, a clustering mechanism wasapplied based on Fig. 4. The cluster was divided based on the angle degree of sensor nodes coordinate location to BS. It was intended to categorize the cluster region based on geographical location from BS. Hence, the sensor nodes distribution becomes well organized from the BS point of view.

At the end of this phase, the scattered region of sensor nodes in the network has been established and created a predefined routing table management. The output of the FSM setup phase is shown in Fig. 5. The figure is shown the network with three clusters.

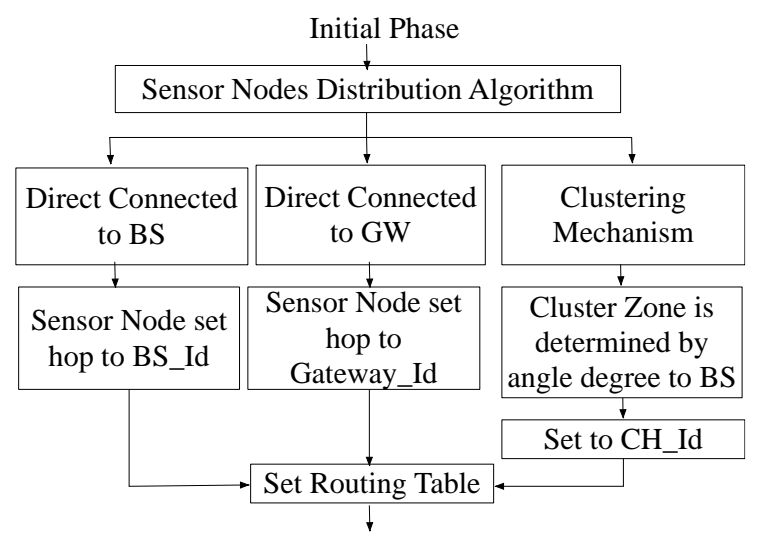

Cluster Head Selection Phase

Figure. 4 Setup Phase Flowchart

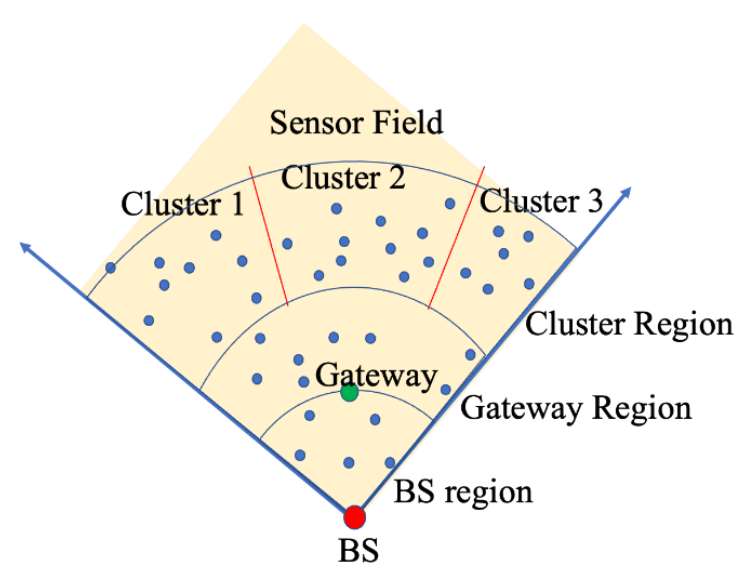

Figure. 5 FSM setup phase network establishment

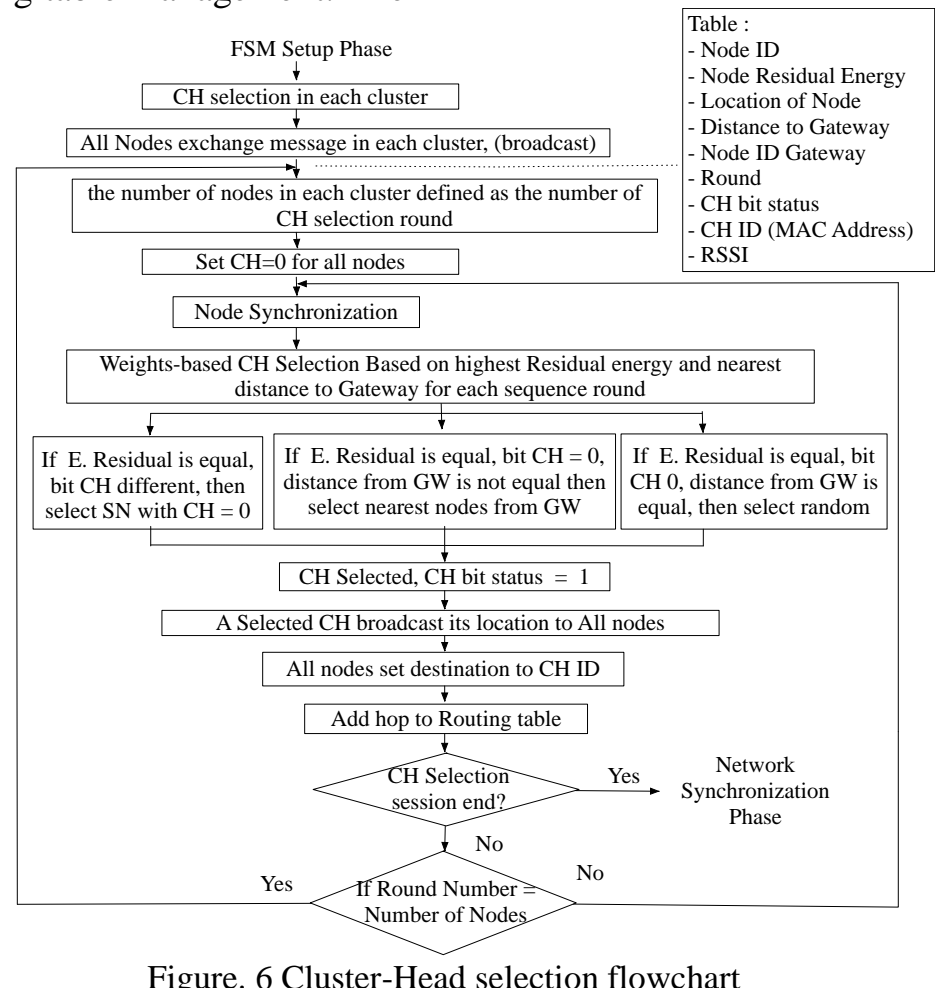




\subsubsection{FSM cluster-head selection phase}

This phase is where each cluster determines its cluster head. Regarding this condition, all sensor nodes in the cluster transmits their data to the $\mathrm{CH}$. Based on Fig. 6, each member node initially exchanges messages, including distance to $\mathrm{GW}$ and energy residual among the cluster members. The $\mathrm{CH}$ will be selected based on weights-based parameter which is consisted of the nearest distance to GW and maximum residual energy. Therefore, in this state, the first selected $\mathrm{CH}$ is determined without probability as stated in Q.Nadeem and N.Singh, but measured by the nearest distance as the least power consumption and the maximum residual energy. The random selected $\mathrm{CH}$ only occurs when all parameters are in the same condition.

Firefly synchronization in the node cluster level is applied in this phase. It is intended to synchronize all the sensor nodes in the cluster, and schedule the data transmission to $\mathrm{CH}$. Then, every time the $\mathrm{CH}$ has sent aggregation data, a new $\mathrm{CH}$ selection will be conducted, and the previously selected $\mathrm{CH}$ becomes a member node. The $\mathrm{CH}$ bit status is used as a token for the selected $\mathrm{CH}$. $\mathrm{CH}$ bit status $=1$ when the sensor node has been selected as $\mathrm{CH}$, and return to zero when entering the new rounds.

Next, $\mathrm{CH}$ aggregates and transmits data packets to $\mathrm{GW}$. Then, GW transmits data packets to $\mathrm{BS}$. $\mathrm{CH}$ data transmission will be scheduled using firefly synchronization in the network level.

\subsubsection{FSM synchronization phase}

This phase is the state where the Firefly algorithm is adapted into the established network. In general, the WSN sensor node's scheduling is managed using TDMA schedule. In TDMA, there is no guarantee that TDMA time slot has the same clock as the sensor node clock. When a node gets a time slot from TDMA, a delay occurs between the TDMA clock and sensor node clock. These clock differences can potentially cause time delays. Time delay increases sensor nodes of power consumption. Therefore, synchronization should be considered in the design network. Synchronization is intended to minimize the waiting time for each sensor node. In transmission data, minimizing waiting time does not directly correlate with a routing protocol; however, it correlates with saving energy consumption.

Firefly synchronization [23] was conducted at the sensor level in the cluster as node synchronization. This is applied in the $\mathrm{CH}$ selection phase. Since there were sensor nodes on the network that are directly
FSM CH Selection Phase

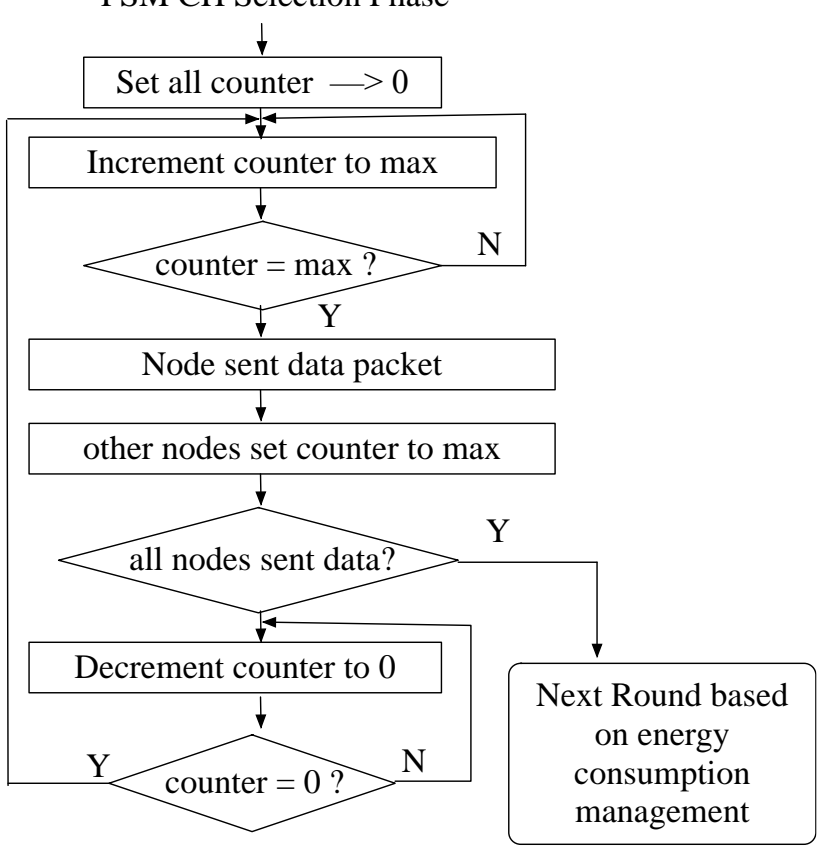

Figure. 7 Node and network synchronization flowchart in one round

connected to GW and BS, Firefly was also adapted for the network level.

As shown in Fig. 7, each node is started to send the data packets in the data transmission process. The firefly set the clock-counter period to synchronize among sensor nodes. The maximum value of the counter is determined by the maximum number of the member sensor nodes in each cluster. The clockcounter then will be incremented or decremented periodically. The clock-counter in each node starts to count in the different starting time. The first sensor node that reaches the maximum clock-counter value will transmit the data packets. In this state, the other sensor nodes are set up their clock-counter to the maximum value.

For all sensor nodes with maximum clockcounter value, they start to decrement the clockcounter value until zero. Furthermore, when the clock-counter reaches the zero value, the clockcounter started to increment again. When all sensor nodes have sent their data packets, all sensors will sleep (steady-state phase) until the next round.

When $\mathrm{CHs}$ are transmitting the data packet to the gateway, they are also using firefly synchronization for the network level. The synchronization is arranged between the $\mathrm{CHs}$ and other nodes that are directly connected to $\mathrm{GW}$. The process of synchronization between $\mathrm{CHs}$ and $\mathrm{GW}$ also refer to in the flowchart in Fig. 7. To have the same period with other GW and to avoid the data collision, the clock-counter must be set based on the maximum number of the member clusters from a particular $\mathrm{CH}$ 
and the member sensor nodes that directly connect to GW. Thus, by adopting firefly synchronization at the node and network level, it is expected to reduce energy consumption. In addition, firefly indirectly assists the routing protocol in terms of scheduling as well as TDMA in GEAR protocol.

\subsubsection{FSM steady-state phase}

The synchronization phase takes many iterations. When all sensor nodes are synchronized, the network is in the steady-state phase. In this phase, all sensor nodes transmit data packets to the $\mathrm{CHs}$. $\mathrm{CHs}$ collect data packets from member nodes then transmit data packets to GW. GW collect data packets from $\mathrm{CHs}$ and the directly connect sensor node, then transmit the data packets to BS. BS will receive all data packets from the GW and directly connect sensor nodes. All sensor nodes are in sleep mode. The next iteration will be started again at the $\mathrm{CH}$ selection phase based on energy consumption formulation.

\subsection{Energy consumption management}

The radio model applied is First Order Radio [28]. It represents energy dissipation processes such as transmit, receive, and aggregate from each sensor node. The transmitter dissipates more energy than the receiver because the receiver is only electronics circuit that dissipates the energy. The model is shown in Fig. 8.

Based on Fig. 8, $k$ bits in the formula is intended to transmit or receive data packets with distance. The formula is given as Eq. (3), (4) and (5). At Eq. (3), $E_{T X}$ has required energy utilization for packet transmission and influenced by $E_{T x-e l e c}$ sensor nodes hardware and $E_{T x \text {-radio }}$ from sensor node radio hardware. $E_{T x-e l e c}$ is electronic energy that consumed from the devices, while $E_{T x \text {-radio }}$ is energy that consumed by the radio hardware to communicate with other components.

At Eq. (4), the energy transmission is affected by the amount of data packets in bits and distance among devices. Thus, it is written as a function in bits $(k)$ and distance $(d)$. Therefore, $E_{T X}(k, d)$ is the energy transmission that has been affected by bits and distance. While in the receiver section, $E_{R x}$ that is as required energy utilization for packet receiving only affected by bits $(k)$ and $E_{\text {elec }}$ as a receiving energy is formulated in Eq. (5).

$$
\begin{gathered}
E_{T X}=E_{T X-\text { elec }}+E_{T X \text {-radio }} \\
E_{T X}(k, d)=E_{T X-e l e c}(k) \\
+E_{T X-A m p}(k, d)
\end{gathered}
$$

$$
E_{R X}(k)=k \times E_{\text {elec }}
$$

In proposed system, at energy consumption management, the radio model is modified by weights-based parameter $(w)$ that measured by the RSSI distance to energy consumption. Hence, the function of $(k, d)$ in the Eq. (4) will be modified by $(k$, $d(w))$ as seen in Eq. (6).

$$
\begin{aligned}
E_{T X}(k, d(w))= & E_{T X-e l e c}(k) \\
& +E_{T x-a m p}(k, d(w))
\end{aligned}
$$

Based on Eq. (6), it can be seen that the energy transmission has been influenced by weights-based parameter. Thus, $E_{T X}(k, d(w))$ is energy transmission in which there is a weights ratio of energy consumption based on RSSI. Energy aggregation is also calculated in the Eq. (7) at the $\mathrm{CH}$ level.

$$
E_{T X C H}(k, d(w))=E_{T X}(k, d(w))+E_{A g g}
$$

At the $\mathrm{CH}$ level, energy aggregated $\left(E_{\text {Agg }}\right)$ is required as the energy to collect the data packets from member nodes. Thus, as seen in Eq. (7) the energy aggregation will be calculated with energy consumption to send data packets to $\mathrm{GW}$ as the energy that consumed to transmit the data packets by the CHs. The energy consumption sensor node can be analyzed in the Eq. (8) in the following formula.

$$
E_{\text {consumption }}=E_{T X}(k, d(w))
$$

Based on Eq. (8), energy consumption sensor node $\left(E_{\text {consumption }}\right)$ also influenced by weights-based parameters. The same as Eq. (6). Energy consumption is executed by many schemes, such as energy consumption for direct connection to BS and GW, energy consumption for member nodes in the cluster, and energy consumption for $\mathrm{CHs}$ when transmitting the data packet to $\mathrm{GW}$ and receive data from member nodes. The distance parameter at each node varies depending on the sensor node's signal strength, where this parameter determines the distance between the hops.

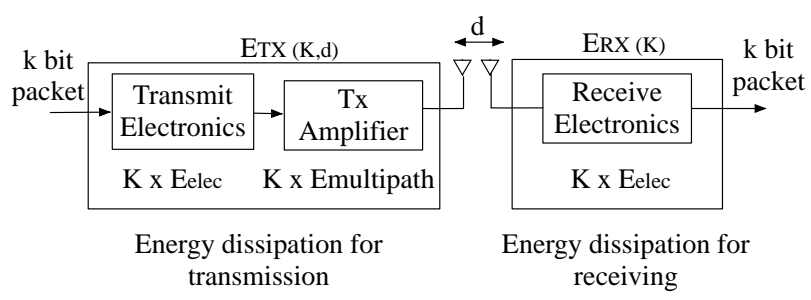

Figure. 8 Radio model 
In this research, residual energy also measured to define whether the node can still transmit the data packets for the next round. Therefore, the residual energy ( $\left.E_{\text {Residual }}\right)$ is formulated in the Eq. (9). Residual energy is formulated by subtraction the initial energy with energy consumption.

$$
E_{\text {Residual }}=E_{\text {Initial }}-E_{\text {Consumption }}
$$

Based on Eq. (9), energy residual is sensor node's remaining energy per each round, while initial energy $\left(E_{\text {Initial }}\right)$ is the initial energy that defined for each sensor nodes in the first round. Energy consumption is the energy required when transmitting data packets. This formula taken as a stated to continue the simulation as long as the remaining energy is greater than energy consumption as shown in Fig. 2.

\section{Result and discussion}

This section is started with setting up the simulation and parameters. Then, determining the sensor node distribution. It is intended in order to define the optimal network model as illegal logging requirements. The optimal network model then is examined on network lifetime, residual energy, and throughput parameters. Besides, at the end of this section, there will a discussion about FSM-GEAR in the illegal logging domain.

\subsection{Setup simulation and parameters}

Running an experiment in a real wide-area of WSN is costly and challenging to implement. According to this condition, the network simulation conducting with testing space area in twodimensional coordinate using MATLAB. The initial assumption is pointed as node are static, homogeneous, all nodes have uniform energy when deployed, BS located at the coordinate $(0.0)$, unique ID in each node and energy consumption transmission in each node various based on the RSSI distance factor. Subsequently, simulation of sensing and monitoring area was run in many iterations. The assumption value on the RSSI distance is applied based on the experimental research field [29]. Throughput, power consumption and network lifetime were calculated in iteration parameters. The results were compared with the previous research and analyzed in the form of a table and graphics.

The simulation was carried out using M-GEAR with TDMA scheduling by Q.Nadeem, and enhanced M-GEAR with TDMA scheduling by N.Singh as a comparison to the proposed Firefly Synchronization Multi-hop protocol (FSM-GEAR). The simulation parameters are listed in Table 1.

Table 1. Simulation parameter

\begin{tabular}{|c|c|c|}
\hline Parameter & Description & Value \\
\hline $\mathrm{N}$ & Number of Sensor nodes & 100 \\
\hline $\mathrm{M} \times \mathrm{M}$ & Network Size & $100 \times 100$ \\
\hline $\mathrm{E}_{\text {initial }}$ & $\begin{array}{c}\text { Energy generated in the } \\
\text { beginning of simulation (J/bit) }\end{array}$ & 0.5 \\
\hline $\mathrm{E}_{\mathrm{elec}}$ & $\begin{array}{c}\text { Energy consumed by a sensor } \\
\text { node (nJ/bit) }\end{array}$ & 100 \\
\hline $\mathrm{E}_{\mathrm{Agg}}$ & $\begin{array}{c}\text { Energy consumed to aggregate } \\
\text { data (nJ/bit) }\end{array}$ & 5 \\
\hline $\mathrm{E}_{\mathrm{TX}}$ & $\begin{array}{c}\text { Energy consumed by } \\
\text { transmitting data (nJ/bit) }\end{array}$ & 50 \\
\hline $\mathrm{E}_{\mathrm{RX}}$ & $\begin{array}{c}\text { Energy consumed by receiving } \\
\text { data (nJ/bit) }\end{array}$ & 50 \\
\hline $\mathrm{E}_{\mathrm{radio}}$ & $\begin{array}{c}\text { Energy consumed by the radio } \\
\text { transmission (pJ) }\end{array}$ & 100 \\
\hline $\mathrm{k}$ & Data Packets (bit) & 4000 \\
\hline
\end{tabular}

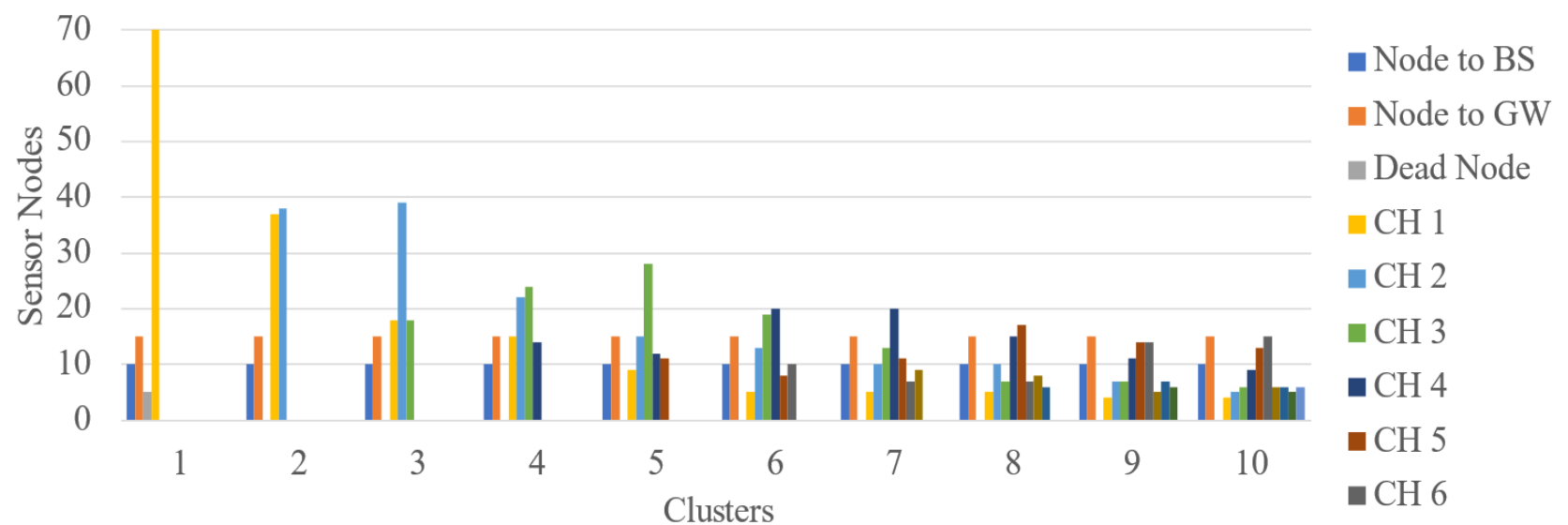

Figure. 9 Node distribution based on the number of clusters 


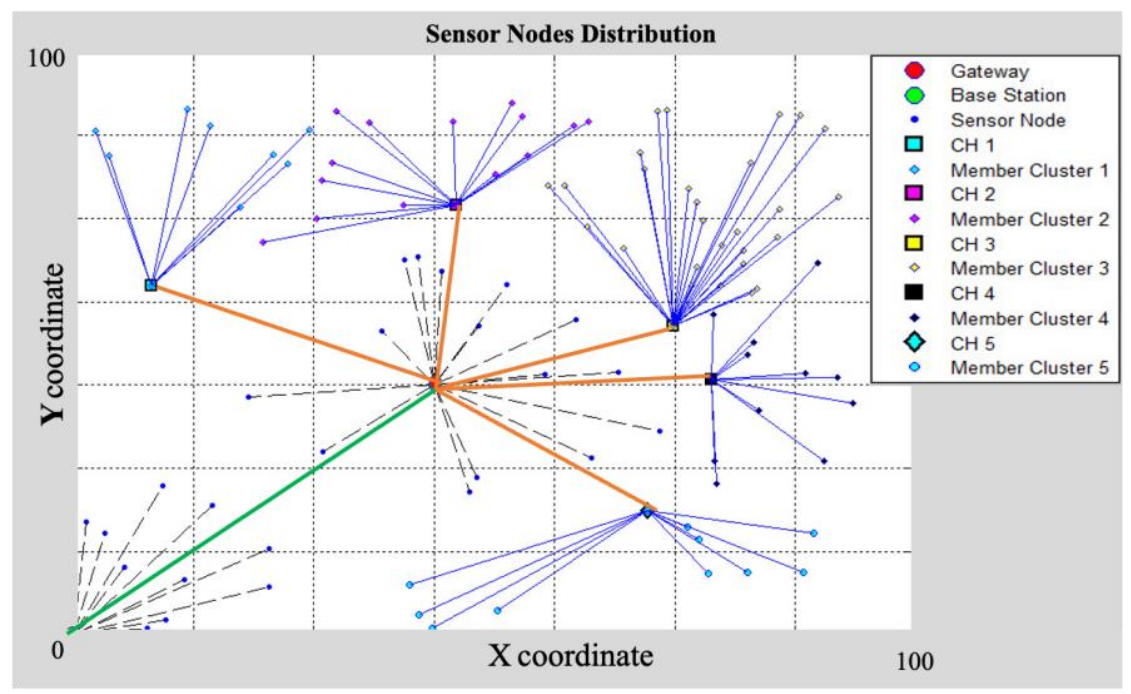

Figure. 10 Network configuration with five clusters

\subsection{Sensor node distribution}

In this section, the experiments conducted sensor nodes distribution based on regions in the network, with various number of CHs. Fig. 9 is shown the configuration of the distribution sensor based on Eq. (1) and (2).

In Fig. 9, the number of sensor nodes connected to $\mathrm{BS}$ and $\mathrm{GW}$ were static. 10 sensor nodes were directly connected to BS, while 15 sensor nodes were directly connected to GW. The remaining sensor nodes are defined as clusters.

The graph showed the number of sensor nodes distribution that varies in each cluster. In cluster_1, all the remaining sensor nodes formed only one cluster. Unfortunately, in this state, there were five dead nodes in the cluster. However, dead nodes did not exist in cluster_2 to cluster_10.

A fairly even distribution sensor nodes were seen in cluster 5 to cluster 10 , while the number of sensor nodes varied in cluster_5 and cluster_6. All sensor node formations in the cluster will be tested for energy consumption in the next test. The network with five clusters configuration is outlined in Fig. 10.

\subsection{Parameters examination}

In this section, the FSM-GEAR protocol will be examined using five clusters network configuration. The tests were conducted on three types of protocol, M-GEAR, enhanced M-GEAR, and proposed FSMGEAR. This proposed algorithm aimed to maximize the throughput, synchronize the network, and save more energy consumption. It simulated several scenarios to proceed with information about network lifetime, energy residual, throughput, and optimum model.

\subsubsection{Optimum model}

This scenario is to measure the number of optimum clusters resulted as the best performance in the proposed system. The parameter to be measured is the number of alive nodes versus the number of cluster or the number of clusters versus number of round or time. The graph can be seen in Fig. 11.

Based on the graph in Fig. 11, the distribution of the minimum number of alive nodes in each cluster configuration is depicted in blue lines. The greater the minimum alive node can indicate the longer the lifetime of a cluster. Meanwhile, the red line represents the distribution of the standard deviation of the number of alive nodes in each cluster. The standard deviation value indicated the differences in the number of alive nodes between nodes in a cluster. The distribution of the minimum alive node values and the distribution of standard deviation values were intersecting at the first point between five and six clusters. Hence, the network configuration with fiveclusters represents a combination minimum number of alive nodes and the optimal standard deviation.

The distributed optimal model for illegal logging WSN is represented, as shown in Figure 12. The network is represented the distributed of five-clusters, one base station area, and one gateway area.

\subsubsection{Network lifetime}

Network lifetime is the interval time during operation from the beginning of sensing process until the energy are depleted in the networks. This scenario is measured the number of alive nodes until energy depleted. The parameter to be compared is the number of alive nodes versus time or round. The result can be seen in Fig. 13. 


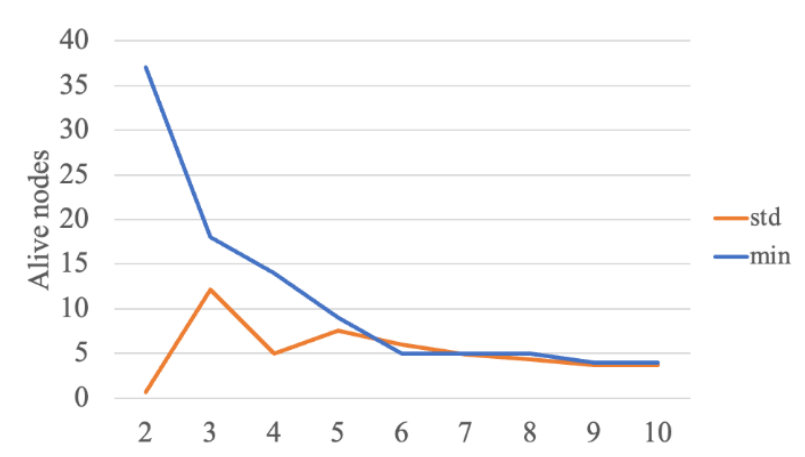

Figure. 11 Optimum model graph

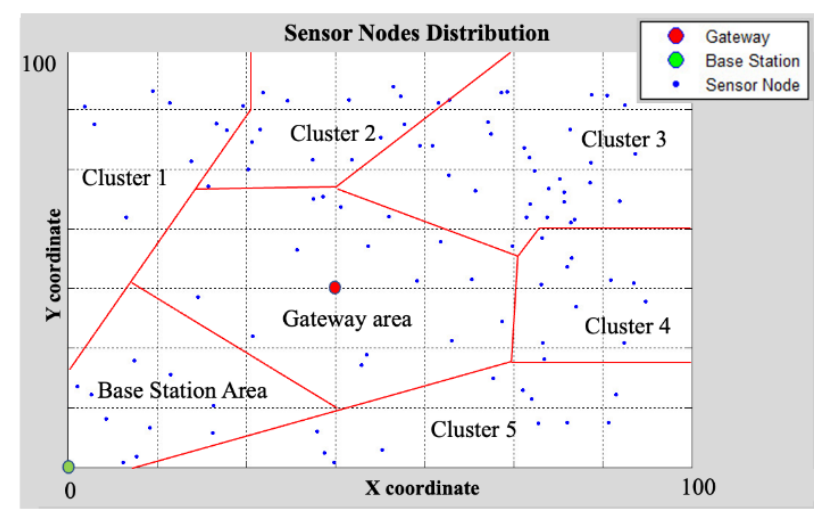

Figure. 12 Distributed sensor nodes on five clusters configuration

Based on Fig. 13, M-GEAR protocol resulted the number of alive nodes at the beginning of round until 82 rounds was constant. It means that there was no dead node until round 82. Since round 83, M-GEAR protocol generated the same number of dead nodes up to round 139. Then, dead nodes became increased or depleted energy at round 249. Meanwhile, Enhanced M-GEAR protocol started had dead nodes at round 93. It was 10 rounds longer than M-GEAR protocol, and totally depleted at round 252. Whereas in FSMGEAR protocol, dead nodes started occur since round 247 , and all nodes became dead nodes at round 280.

In the graph, it can be seen that M-GEAR and enhanced M-GEAR seem to have the same characteristics but differences. Enhanced M-GEAR has better characteristics than M-GEAR. The difference lies in the distance that applied in the initial network and the maximum energy on the $\mathrm{CH}$ selection. Meanwhile, FSM-GEAR protocol is showed better characteristics than M-GEAR and Enhanced M-GEAR protocols. This is due to the consideration of firefly synchronization and weightsbased parameters according to nearest distance and maximum residual energy which is implemented in $\mathrm{CH}$ selection and energy consumption management.

Based on these differences, it can be concluded that the network lifetime can be extended by using
Firefly Synchronization and weights-based parameter. Even though it only expanded 31 rounds, Firefly synchronization and weights-based parameter can prolong the network lifetime.

\subsubsection{Residual energy}

Residual energy is the remaining energy in sensor nodes after each round. This scenario is also implemented to evaluate network performance. The parameter to be measured is remaining energy versus rounds or time.

In Fig. 14, it can be seen that all protocols have decreased in residual energy, along with increasing rounds in data communication cycle between nodes in the network. However, the energy drop in MGEAR and Enhanced M-GEAR protocols were greater than in FSM-GEAR protocol. Therefore, MGEAR and enhanced M-GEAR protocol were faster to reach the state where all nodes were in a dead node state and no longer can transmit the data packets. According to the result, FSM-GEAR protocol is proven to be able to extend the network lifetime.

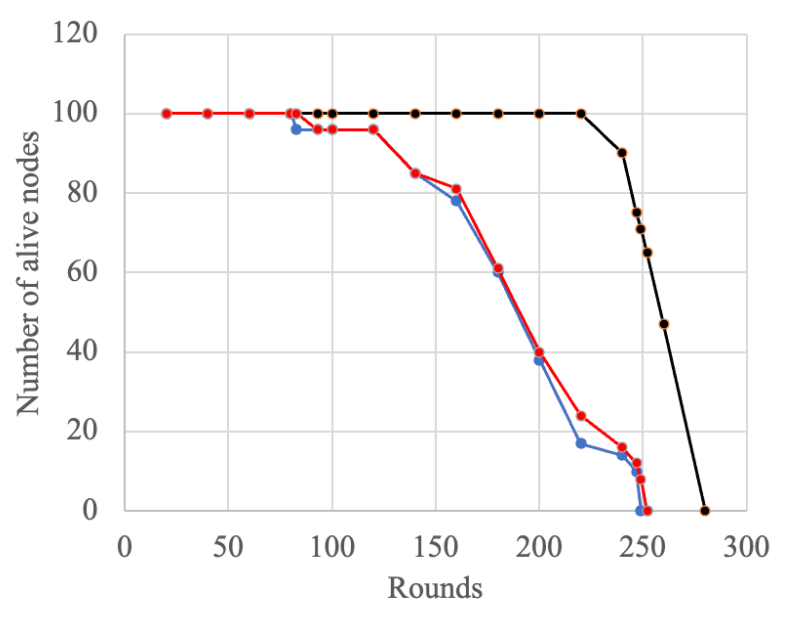

--M-GEAR -•-FSM-GEAR -•-Enhanced M-GEAR

Figure. 13 Network lifetime comparison between MGEAR, enhanced M-GEAR, and FSM-GEAR

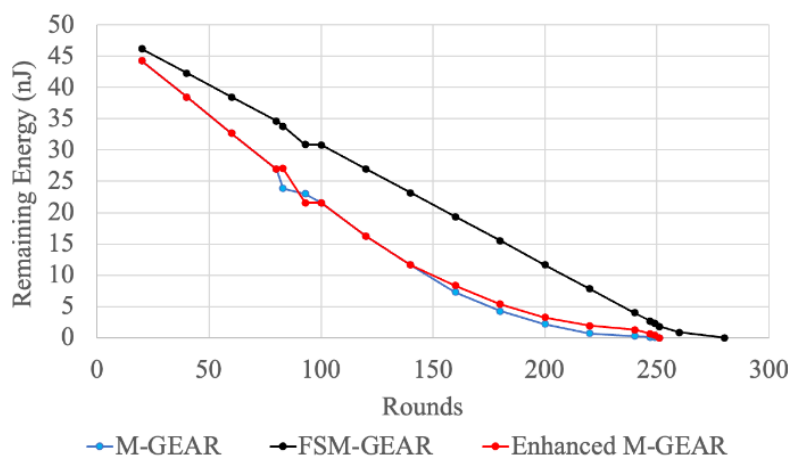

Figure. 14 Residual energy comparison between MGEAR and FSM-GEAR 


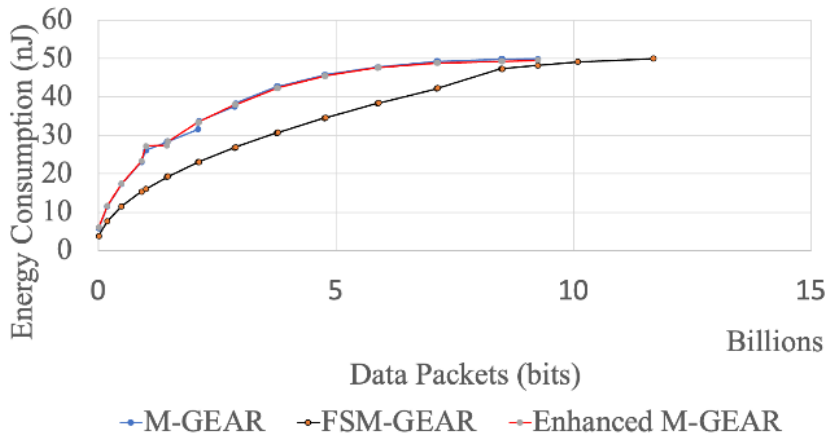

Figure. 15 Throughput comparison between M-GEAR and FSM-GEAR

\subsubsection{Throughput}

This parameter measured how many data packets were transferred from the sensor node to BS. This scenario evaluated the network performance, as well as ensured the energy balance by $\mathrm{CH}$. The parameter to be compared is the number of data packets received by BS versus energy consumption in each round. Hence, the greater number of packets received at the BS, represented, the longer the network lifetime. The result can be seen in Fig. 15.

Based on Fig. 15, there were less data packets sent by M-GEAR and enhanced M-GEAR protocol than FSM-GEAR protocol. Weights-based FSMGEAR protocol was $1.393 \mathrm{MB}$, M-GEAR protocol was $1.103 \mathrm{MB}$ and enhanced M-GEAR 1.158 MB. This is because the network lifetime on weightsbased FSM-GEAR was longer than the two comparison protocols.

\subsection{FSM-GEAR protocol performance}

Based on the test result, a FSM-GEAR protocol performance can be discussed. FSM-GEAR protocol offered better performance than two comparison protocols. The effectiveness of the proposed system was offered by weights-based parameter in FSMGEAR protocol at $\mathrm{CH}$ selection phase, provides more longer network lifetime, fit residual energy, and more data packet delivered to BS. Besides, firefly synchronization acts as fit scheduling to transmit the data packets and reduce the waiting time.

Weights-based FSM-GEAR protocol applied in the forest characteristics offers the solution of forest area energy-efficient surveillance. Thus, weightsbased FSM-GEAR protocol provides a solution for monitoring forest areas from illegal logging. The characteristics of weights-based FSM-GEAR protocol, which are multi-hop and energy-efficient, can provide forest monitoring from illegal logging activities. The multi-hop configuration supports surveillance of vast forest areas with distributed sensor allocation. Appropriate energy-efficient solutions are crucial since the forest area is rural and has an electricity source limitation. Weights-based FSM-GEAR is the proper solution to monitor and control the forest areas' security from illegal logging activities based on these characteristics.

\section{Conclusions}

WSN implementation in illegal logging application requires communication protocol support that is capable for covering the wide-area forest. Considering the area to be covered, an analysis of sensor node distribution is very substantial for the application. The variation distribution of sensor nodes, the limited energy sources on sensor nodes, and the unavailability of power sources in the forest area required an energy-efficient WSN configuration. Weights-based FSM-GEAR protocol was designed to support the WSN's monitoring for illegal logging application. The variations in the distribution of sensor nodes were adapted to the sensor nodes' RSSI signal strength. Weights-based parameter considering the nearest distance to GW and maximum residual energy applied for $\mathrm{CH}$ selection and energy consumption management. Besides, energy limitation was also conducted by energy saving through firefly synchronization algorithms and sensor node clustering distribution.

The five-cluster model network offers an optimal model network to simulate for further analysis. The simulation results showed that weights-based FSMGEAR protocol had better performance than MGEAR and enhanced M-GEAR protocols. Weightsbased FSM-GEAR protocol depleted energy in round 280, while M-GEAR and enhanced M-GEAR protocols are depleted in round 247 and round 252. Meanwhile at the data packets delivered to BS, weights-based FSM-GEAR protocol was $1.393 \mathrm{MB}$, M-GEAR protocol was $1.103 \mathrm{MB}$ and enhanced MGEAR 1.158 MB. At the energy consumption analysis, M-GEAR and enhanced M-GEAR protocols were faster depleted energy than weightsbased FSM-GEAR protocol. According to simulation results, FSM-GEAR provide more effective energy consumption since it can prolong the network lifetime.

For future work, FSM-GEAR protocol offers various further enhancements to the data security aspects of each data transmission from the sensor node to the $\mathrm{CH}$, from the $\mathrm{CH}$ to $\mathrm{GW}$, and from $\mathrm{GW}$ to BS. Data security aspects can be in the form of adding security bytes to the data packet arrangement or integration with certain security protocols. Another development that can be enhanced is 
providing predictive capabilities in the form of dead node prediction, sensor distribution prediction, and energy residual prediction as one of the determinant factors from cluster head selection. In addition, it is also developing how to optimize the last remaining energy in each sensor nodes.

\section{Conflicts of Interest}

The authors declare no conflict of interest.

\section{Author Contributions}

The contributions of authors are as follows: conceptualization, validation, Giva Andriana Mutiara and Othman Mohd; methodology, software, formal analysis, investigation, resources, data curation, writing - original draft preparation, project administration, funding acquisition, Giva Andriana Mutiara; writing - review and editing, Giva Andriana Mutiara, Othman Mohd; supervision, Othman Mohd, Nanna Suryana Herman, Ahmad Naim Che Pee.

\section{Acknowledgments}

I would like to express my appreciation to Center for Advanced Computing Technology (C-ACT), Faculty of Information and Communication Technology, Universiti Teknikal Malaysia Melaka (UTeM) and thank you to Embedded and Network System Research Group Laboratory of Telkom University, Bandung, Indonesia for supported this research.

\section{References}

[1] C. Buratti, A. Conti, D. Dardari, and R. Verdone, "An Overview On Wireless Sensor Networks Technology And Evolution", Sensors, Vol. 9, No. 9, pp. 6869-6896, 2009.

[2] F. M. Al-turjman, H. S. Hassanein, and M. Ibnkahla, "Towards Prolonged Lifetime For Deployed WSNs In Outdoor Environment Monitoring", Ad Hoc Networks, Vol.24, No. Part A, pp. 172-185, 2014.

[3] M. Elshrkawey, S. M. Elsherif, and M. E. Wahed, "An Enhancement Approach for Reducing the Energy Consumption in Wireless Sensor Networks", Journal of King Saud University. - Computer Information Sciences, Vol. 30, No. 2, pp. 259-267, 2018.

[4] I. F. Akyildiz, and M. C. Vuran, Wireless Sensor Network. John Wiley and Sons, Ltd, 2010.

[5] S. Chakravarty, S. K. Ghosh, C. P. Suresh, A. N. Dey, and G. Shukla, "Deforestation: Causes, Effects and Control Strategies", Global
Perspectives on Sustainable Forest Management, InTech, pp. 3-29, 2012.

[6] J. Saunders, Illegal Logging and Related Trade The Response in Lao PDR. A Chatham House Assessment, 2014.

[7] G. A. Mutiara, N. Suryana, and O. Mohd, "Wireless Sensor Network For Illegal Logging Application: A Systematic Literature Review", Journal of Theoritical and Applied Information Technology., Vol. 97, No. 1, pp. 302-313, 2019.

[8] L. Czúni, and P. Z. Varga, "Lightweight Acoustic Detection of Logging in Wireless Sensor Networks", In: Proc. of the International Conference on Digital Information, Networking, and Wireless Communications, pp. 120-125, 2014.

[9] W. B. Heinzelman, A. P. Chandrakasan, and H. Balakrishnan, "An Application-Specific Protocol Architecture For Wireless Microsensor Networks", IEEE Transactions on Wireless Communication., Vol. 1, No. 4, pp. 660-670, 2002.

[10] K. Keerthana, C. Padmavathy, and M. Rojaramani, "A Survey on Minimizing Delay and Maximizing Lifetime in Wireless Sensor Networks", International Journal of Research in Computer Applications and Robotics, Vol. 4, No. 3, pp. 55-58, 2016.

[11] H. Yetgin, K. T. K. Cheung, M. El-Hajjar, and L. Hanzo, "A Survey of Network Lifetime Maximization Techniques in Wireless Sensor Networks", IEEE Communications Surveys and Tutorials, Vol. 19, No. 2, pp. 828-854, 2017.

[12] W. Dargie, and C. Poellabauer, Fundamentals of Wireless Sensor Networks : Theory and Practice, Vol. 136, No. 1. John Wiley \& Sons, Inc., Hoboken New Jersey, 2010.

[13] G. Kaur, "Enhanced M-Gear Protocol for Lifetime Enhancement in Wireless Clustering System", International Journal of Computer Applications, Vol. 147, No. 14, pp. 30-34, 2016.

[14] D. D. Geetha, and N. Tabassum, "A Survey On Clock Synchronization Protocols In Wireless Sensor Networks", In: Proc. International Conference on Smart Technologies for Smart Nation, SmartTechCon pp. 504-509, 2017.

[15] Q. Nadeem, M. B. Rasheed, N. Javaid, Z. A. Khan, Y. Maqsood, and A. Din, "M-GEAR: Gateway-based energy-aware multi-hop routing protocol for WSNs", In: Proc. of 8th Int. Conf. on Broadband and Wireless Computing Communication and Applications. BWCCA 2013, pp. 164-169, 2013.

[16] N. C. Bantayan, "Integrating Radio-Frequency Identification ( RFID ) and Geomatics Towards 
Precision Forestry Integrating Radio-Frequency Identification ( RFID )", Wulfenia Journal., Vol. 22, No.5, pp. 241-252, 2015.

[17] J. Häkli, K. Jaakkola, P. Pursula, M. Huusko, and K. Nummila, "UHF RFID Based Tracking of Logs in the Forest Industry", In: Proc. of IEEE International Conference on RFID, $\mathrm{pp}$. 245-251, 2010.

[18] S. Wang, K. Wang, X. Wang, and Z. Liu, “A Novel Illegal Logging Monitoring System Based On WSN", Advanced Materials Research, Vol. 518-523, pp. 1417-1421, 2012.

[19] F. M. Al-turjman, H. S. Hassanein, and M. A. Ibnkahla, "Connectivity Optimization for Wireless Sensor Networks Applied to Forest Monitoring", IEEE Communication Society, pp. 1-6, 2009.

[20] Y. Yu, R. Govindan, and D. Estrin, Geographical and Energy Aware Routing: A Recursive Data Dissemination Protocol for Wireless Sensor Networks, UCLA Computer Science Department Technical Report, Vol. 463, pp. 1-11, 2001.

[21] J. Zheng, and A. Jamalipour, Wireless Sensor Networks, John Willey and Sons. Inc, 2017.

[22] N. Singh, and N. Mahajan, "The Enhanced MGEAR Protocol for Wireless Sensor Network LifeTime", International Journal of Computer Science and Technology, Vol. 9, No. 4, pp. 98100, 2018.

[23] M. Chovanec, J. Púchyová, M. Hudik, and M. Kochláň, "Universal Synchronization Algorithm for Wireless Sensor Networks"FUSA algorithm", In: Proc. of the 2014 Federated Conference on Computer Science and Information System, Vol. 2, pp. 1001-1007, 2014.

[24] N. Jabeur, "A Firefly-Inspired Micro and Macro Clustering Approach for Wireless Sensor Networks", Procedia - Procedia Computer Sciences, Vol. 98, pp. 132-139, 2016.

[25]E. S. Kumar, S. M. Kusuma, and B. P. V. Kumar, "Fire-LEACH : A Novel Clustering Protocol for Wireless Sensor Networks based on Firefly Algorithm", International Journal of Computer Science: Theory and Application, Vol.1, No.1, pp. 12-17, 2015.

[26] M. Baskaran and C. Sadagopan, "Synchronous Firefly Algorithm for Cluster Head Selection in WSN", Scientific World Journal, Vol. 2015, pp. $1-7,2015$.

[27] S. Halder, and A. Ghosal, "Is Sensor Deployment Using Gaussian Distribution Energy Balanced", In: Proc. of International Conference on Algorithms and Architectures for
Parallel Processing, TCE Chem. Eng., No. 891, pp. 52-54, 2015.

[28] P. K. Batra and K. Kant, "LEACH-MAC: A New Cluster Head Selection Algorithm for Wireless Sensor Networks", Wireless Networks, Vol. 22, No. 1, pp. 49-60, 2016.

[29] G. A. Mutiara, N. S. Herman, and O. Mohd, "Using Long-Range Wireless Sensor Network To Track The Illegal Cutting Log", Applied Sciences, Vol. 10, No. 19, pp. 1-17, 2020. 